\title{
Method of determining the effective surface area of a rigid body under wind disturbances
}

Received: 4 May 2020 / Accepted: 10 August 2020 / Published online: 20 August 2020

(C) The Author(s) 2020

\begin{abstract}
This work proposes a method for determining the effective surface area of a rigid body induced by the wind. The proposed method assumes a variable surface effective area on which the wind acts. To determine the effective surface parameter, the dependence of normal vectors and the position change of individual vertices of the solid body were used. Cardan angles and its derivatives were used to determine the rigid body orientation in space. The free motion of the rigid body was analysed for two variants: influence of wind force and forced motion. During calculations, various cases were analysed differing in terms of wind speed and direction. A comparison of the developed method with the constant and projected surface area was presented. Various acceptable cases in which the surface area approximation is permissible were also shown. The proposed method can be used to calculate the wind load acting on a load of transport devices.
\end{abstract}

Keywords Variability wind area $\cdot$ Wind pressure $\cdot$ Rigid body motion $\cdot$ Cardan angles

\section{Introduction}

The motion of a rigid body is one of the elementary sections of classical mechanics. The description of such motion consists of the equations of translational and rotational motion. In the literature, one can rarely find works which consider the impact of external forces in the theoretical model. An example of such a force is the wind pressure which can cause significant changes in the trajectory of such a body motion trajectory.

Nowadays, works related to the subject of wind load effect on structures can be divided into two categories: devices (structures in motion) [1,7,11,12,15,17,24] and motionless structures [8,10,22,23].

Articles related to structures in motion generally cover issues related to the devices most exposed to the wind: ships [1,17,24], flying robots [11] or cranes [12,15]. In these works, however, the parameter of effective surface area is not taken into account. In general, the constant maximum area on which wind acts is assumed. A common method is also to include the projected surface only at the initial stage of calculations. In [1], the impact of various loading configurations on the behaviour of a container ship under wind load was presented. Various cases of wind pressure were analysed, causing a change in the determined course of the ship. A fixed projected area for two directions (longitudinal and transverse) was adopted in wind calculations. The obtained results were verified using the wind tunnel. The work [17] concerned numerical simulation of the container ship aerodynamics. Computational fluid dynamics (CFD) was used to calculate mass, energy and

D. Cekus · P. Kwiatoń $(\bowtie)$

Department of Mechanics and Machine Design Fundamentals, Czestochowa University of Technology, Dabrowskiego 69, 42-201

Czestochowa, Poland

E-mail: kwiaton@imipkm.pcz.pl

D. Cekus

E-mail: cekus@imipkm.pcz.pl 
momentum conservation equations. Numerical calculations were carried out in the ANSYS CFX software. During the simulation, the $k-\varepsilon$ turbulence model was adopted. In [24], author proposed a new method of wind impact calculation for the container ship. The proposed method can be used at the design stage in which not all dimensions have been yet specified. The numerical and experimental results were presented as graphs of additional resistance, drift moment and transfer drift force. Gust disturbance control method for QUAVs flying robot was shown in [11]. Unknown time-varying payload influence on dynamics of QUAVs was also analysed. The flying unit was assumed as an ideal rigid body. The influence of wind on vehicle behaviour was divided into two stages: momentum drag and disturbance. A new PPC adaptation strategy for better trajectory control has been proposed as the control system. The influence of wind on the dynamics of payload carried by a mobile crane was presented in [12]. Using the feedback controller, a control system was proposed to minimize the effects of gusts. The results in the residual amplitude form were compared with experimental tests performed on a small-scale model. The dynamic analysis of the tower crane taking into account wind load was described in [15]. In the simulation model, wind load was taken into account according to Bernoulli's formula and Davenport's spectrum of wind speed. In addition, the parameters for determining the crane stability were calculated. In the paper [7], the usage of CAE program (SolidWorks) for wind turbine simulation tests was presented. In the calculation model, the turbine blade surface was projected for the case in which the wind blows perpendicular to the axis of rotation of the turbine. The wind force was calculated using the formula for aerodynamic drag force.

Research on the influence of wind load on motionless structures generally focuses on the study of building environment [10,22,23] and related elements [8]. In this type of research, computational fluid dynamics (CFD) software is the most commonly used method, which allows to determine the aerodynamic parameters of the tested structures. Due to lack of motion, there is only a need to project the surface affected by the wind. However, when a wind with variable direction is analysed, the effective area can be changed, which can cause large variations in results. Numerical studies of the wind load on the roof of a sports building were presented in [10]. For numerical analysis, CFD method with the $k-\varepsilon$ turbulent solver was used. Shape factor was analysed with regard to the wind field environment. For each of the stadium's roof region, the maximum wind shape factor was determined. The influence of the roof shape and its slope on wind pressure distribution was also analysed in [22]. Variability of wind direction was also taken into account in the simulation model. The results were presented in the form of pressure distribution outlines and compared with data obtained during experimental tests. A new approach for determining wind loads for low-rise buildings was proposed in [23]. This approach includes the quasi-steady theory model which consists of both elevation angle and instantaneous wind azimuth. Calculations of the surface pressure parameter of a building included longitudinal, transverse and vertical directions of wind velocity as well as standard pressure coefficients. The obtained statistical results were verified with the experiment. The work [8] concerns the analysis of photovoltaic panel (PV array) which is most often installed on the roofs of buildings. In this work, experimental tests were carried out for various wind incidence angles. The lift coefficient was also calculated for each of these angles.

This paper proposes a new method for determining the effective wind area of a cuboidal rigid body. The theoretical model takes into account the variability of wind direction due to applied Cardan angles. The simulation model allows to track the behaviour of the body, its orientation and positioning, during the free motion. The real value of the wind force for a given body position is also determined.

\section{Methods}

Generally, the wind force acting on the structure is included using the drag force formula, which can be written as $[6,15,17]$ :

$$
\mathbf{F}_{\mathbf{w}}=\frac{1}{2} \rho A_{w} C_{D} \mathbf{V}_{\mathbf{w}}{ }^{2}
$$

or at the design stage, when a constant value of air density $\rho$ is assumed [13]:

$$
\mathbf{F}_{\mathbf{w}}=0.625 A_{w} C_{D} \mathbf{V}_{\mathbf{w}}{ }^{2},
$$

where $\mathbf{F}_{\mathbf{w}}$-wind force vector $(\mathrm{N}), A_{w}$-cross-sectional area (wind area) $\left(\mathrm{m}^{2}\right), \mathbf{V}_{\mathbf{w}}$-wind velocity $(\mathrm{m} / \mathrm{s})$, and $C_{D}$-dimensionless drag coefficient.

In most cases, the wind area $A_{w}$ (Eqs. 1,2) is generally adopted as a constant. To determine the parameter of variable surface area affected by the wind $A_{e w}$ (so-called wind surface or effective surface), the algebraic 


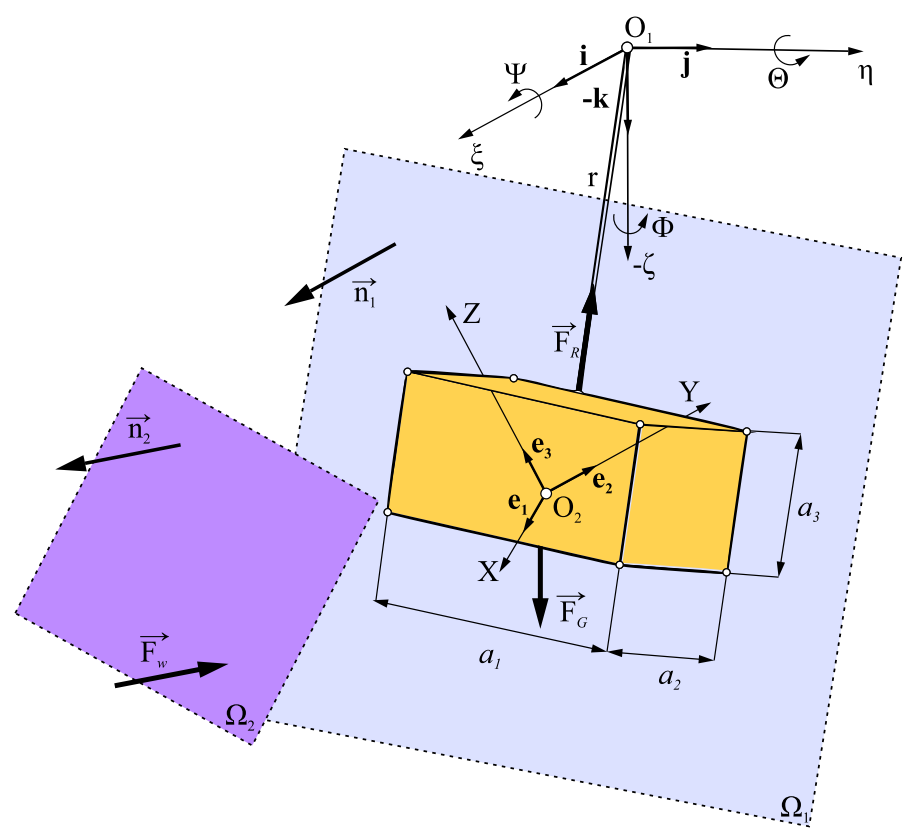

Fig. 1 Dynamic model of a rigid body motion

relation of normal vectors and vertices of a rigid body can be used. Analysing three selected vertices of a rigid body with coordinates:

$$
\mathbf{P}_{\mathbf{1}}=\left(\xi_{1}, \eta_{1}, \zeta_{1}\right), \quad \mathbf{P}_{\mathbf{2}}=\left(\xi_{2}, \eta_{2}, \zeta_{2}\right), \quad \mathbf{P}_{\mathbf{3}}=\left(\xi_{3}, \eta_{3}, \zeta_{3}\right)
$$

one can determine the plane which passes through these vertices. Equation of a such plane can be presented in the form [6]:

$$
\left|\begin{array}{ccc}
X-\xi_{1} & \eta-\eta_{1} & Z-\zeta_{1} \\
\xi_{2}-\xi_{1} & \eta_{2}-\eta_{1} & \zeta_{2}-\zeta_{1} \\
\xi_{3}-\xi_{1} & \eta_{3}-\eta_{1} & \zeta_{3}-\zeta_{1}
\end{array}\right|=0
$$

or in the form:

$$
\left|\begin{array}{llll}
X & Y & Z & 1 \\
\xi_{1} & \xi_{2} & \xi_{3} & 1 \\
\eta_{1} & \eta_{2} & \eta_{3} & 1 \\
\zeta_{1} & \zeta_{2} & \zeta_{3} & 1
\end{array}\right|=0
$$

The general equation parameters of this plane [21]:

$$
\alpha X+\beta Y+\gamma Z+\psi=0
$$

can be determined using formula:

$$
[\alpha, \beta, \gamma]=\left(\mathbf{P}_{\mathbf{2}}-\mathbf{P}_{1}\right) \times\left(\mathbf{P}_{3}-\mathbf{P}_{1}\right) .
$$

Taking into account the plane parameters (Eq. 6), normal vector of a such plane can be depicted as $[6,18]$ :

$$
\mathbf{n}_{1}=\left[\begin{array}{c}
\lambda \alpha \\
\lambda \beta \\
\lambda \gamma
\end{array}\right]
$$

where $\lambda \in \mathbb{R} \backslash 0$. 
Specifying the normal vector $\left(\mathbf{n}_{2}\right)$ of the plane, in which wind blows, one can measure the angle between analysed normal vectors using the formula:

$$
\theta_{i}=\arccos \frac{\mathbf{n}_{\mathbf{1}} \circ \mathbf{n}_{\mathbf{2}}}{\left|\mathbf{n}_{\mathbf{1}}\right| \mid \mathbf{n}_{\mathbf{2}}}, \quad i=1,2,3 .
$$

Finally, the effective wind surface parameter $A_{e w}$ can be represented as the relation [6]:

$$
A_{\text {ew }}=a_{1} a_{2} \cos \theta_{1}+a_{1} a_{3} \cos \theta_{2}+a_{2} a_{3} \cos \theta_{3},
$$

where $a_{1}, a_{2}, a_{3}$-dimensions of the rigid body (m), and $\theta_{1}, \theta_{2}, \theta_{3}$-angles between the normal vectors of particular surfaces (rad).

In order to determine the coordinates of individual vertices of the analysed rigid body, Cardan angles were used. For this purpose, the cosine matrix for elementary rotations had to be determined, which can be presented as $[3,16]$ :

- for $\mathrm{X}$ axis:

$$
\mathbf{R}_{\mathbf{X}}=\left[\begin{array}{ccc}
1 & 0 & 0 \\
0 & \cos \Psi & -\sin \Psi \\
0 & \sin \Psi & \cos \Psi
\end{array}\right]
$$

- for Y axis:

$$
\mathbf{R}_{\mathbf{Y}}=\left[\begin{array}{ccc}
\cos \Theta & 0 & \sin \Theta \\
0 & 1 & 0 \\
-\sin \Theta & 0 & \cos \Theta
\end{array}\right]
$$

- for Z axis:

$$
\mathbf{R}_{\mathbf{Z}}=\left[\begin{array}{ccc}
\cos \Phi & -\sin \Phi & 0 \\
\sin \Phi & \cos \Phi & 0 \\
0 & 0 & 1
\end{array}\right]
$$

The matrix of rotation of reference systems can be obtained by vectors of the rotation matrix of individual axes. Thus, the rotation matrix of the system has the form $[9,16]$ :

$$
\begin{aligned}
\mathbf{R} & =\mathbf{R}_{\mathbf{Z}} \mathbf{R}_{\mathbf{Y}} \mathbf{R}_{\mathbf{X}} \\
& =\left[\begin{array}{ccc}
c \Theta c \Phi & -c \Psi s \Phi+c \Phi s \Theta s \Psi & c \Psi c \Phi s \Theta+s \Phi s \Psi \\
c \Theta s \Phi & c \Psi c \Theta+s \Phi s \Theta s \Psi & c \Psi s \Phi s \Theta-c \Phi s \Psi \\
-s \Theta & c \Theta s \Psi & c \Psi c \Phi
\end{array}\right]
\end{aligned}
$$

where the following designations were introduced: $c \Theta-\cos \Theta, s \Theta-\sin \Theta, c \Psi-\cos \Psi, s \Psi-\sin \Psi, c \Phi-$ $\cos \Phi$ and $s \Phi$ - $\sin \Phi$.

The components of the angular velocity vector $\omega$, for the applied Cardan angles, can be depicted as:

$$
\begin{aligned}
& \omega_{1}=\dot{\Psi} \cos \Theta \cos \Phi-\dot{\Theta} \sin \Phi, \\
& \omega_{2}=\dot{\Psi} \cos \Theta \sin \Phi+\dot{\Theta} \cos \Phi, \\
& \omega_{3}=\dot{\Psi} \sin \Theta+\dot{\Phi} .
\end{aligned}
$$

Angular accelerations can be obtained by differentiating the formulas (14):

$$
\epsilon=\frac{\mathrm{d} \omega}{\mathrm{d} t}
$$

and take the form:

$$
\begin{aligned}
\epsilon_{1} & =\ddot{\Psi} \cos \Theta \cos \Phi-\ddot{\Theta} \sin \Phi-\dot{\Psi} \dot{\Theta} \sin \Phi \cos \Theta \\
& -\dot{\Psi} \dot{\Theta} \cos \Phi \sin \Theta-\dot{\Theta} \dot{\Phi} \cos \Phi \\
\epsilon_{2} & =\ddot{\Psi} \cos \Theta \sin \Phi+\ddot{\Theta} \cos \Phi+\dot{\Psi} \dot{\Theta} \cos \Phi \cos \Theta \\
& +\dot{\Psi} \dot{\Theta} \sin \Phi \sin \Theta-\dot{\Theta} \dot{\Phi} \sin \Phi \\
\epsilon_{3} & =-\ddot{\Psi} \sin \Theta+\ddot{\Phi}-\dot{\Psi} \dot{\Theta} \cos \Theta
\end{aligned}
$$


Coordinates of individual vertices can be determined using the formula:

$$
C_{i j}= \pm \frac{1}{2} a_{k} R_{j k}-r_{k} R_{j k}
$$

where $i=1,2,3,4, j=1,2,3, k=1,2,3$ and $C=X, Y, Z$, while \pm sign depends on the numbering and orientation of the vertex in the coordinate system.

System rotation matrix elements are nonlinear functions of position angles. Due to the angular nature, the phenomena of singularities of the orientation can be found in the calculations. These nonlinearities can be observed during transformations of angular velocity and accelerations vectors.

The general equations of rigid body motion, which consist of translational and rotational motion, can be depicted as two vector equations $[4,11,14]$ :

$$
\begin{aligned}
m \mathbf{a}_{\mathbf{m}} & =\mathbf{F}_{\mathbf{G}}+\mathbf{F}_{\mathbf{R}}+\mathbf{F}_{\mathbf{w}}, \\
\frac{\mathrm{d}}{\mathrm{d} t} \mathbf{K}_{\mathbf{m}} & =\mathbf{M},
\end{aligned}
$$

where $m$ is a mass and $\mathbf{F}_{\mathbf{G}}$ is a weight of the rigid body, $\mathbf{F}_{\mathbf{R}}$ is a reaction force in the rope, $\mathbf{M}$ is a resultant moment, $\mathbf{a}_{\mathbf{m}}$ is an acceleration and $\mathbf{K}_{\mathbf{m}}$ is an angular momentum of rigid body mass centre.

Finally, a system of six second-order differential equations is obtained which can be represented in the form $[6,19]$ :

$$
\mathbf{A} \ddot{\gamma}=\mathbf{B}
$$

where $\ddot{\gamma}$ is a vector of unknowns that can be depicted as:

$$
\ddot{\gamma}=\left[\begin{array}{c}
\ddot{\xi} \\
\ddot{\eta} \\
\ddot{\zeta} \\
\ddot{\Psi} \\
\ddot{\Theta} \\
\ddot{\Phi}
\end{array}\right] .
$$

A detailed method of determining rigid body motion parameters was presented in the previous works of the authors $[4,6,16,19]$.

\section{Sample results of performed simulations}

Examples of simulation results are presented for the free motion of the rigid body suspended on a nondeformable rope. The study analysed different variants of the free motion of the rigid body differing by initial position and direction of the wind. The results were presented in the form of changes in parameters of surface area $\left(A_{w}\right)$. The differences in the analysed models are also presented by the percentage variance of these parameters. Numerical simulations were carried out for two variants in various cases (Table 1). In the first variant, all initial conditions were equal to zero, and the rigid body motion was caused only by the wind pressure. In the second variant, apart from the wind pressure, the forced motion of the rigid body in one direction was also analysed.

In order to model the variability of wind pressure direction (Fig. 2), the Fresnel cosine integral was used, which can be depicted in the form [20]:

$$
\alpha_{w}=\alpha_{\max } \int_{0}^{P} \cos \frac{\pi t^{2}}{2} \mathrm{~d} t
$$

Wind speed variability as a time function of gust (Fig. 3) can be introduced in form [2]:

$$
V_{w}=V_{\max } \sin \frac{\pi}{t_{c}} t
$$

The following parameters were adopted during all numerical simulations: 
Table 1 Simulation cases for both variants

\begin{tabular}{lllll}
\hline Case & $\mathrm{A}$ & $\mathrm{B}$ & $\mathrm{C}$ & $\mathrm{D}$ \\
\hline$V_{w}$ & Constant & Variable & Variable & Constant \\
$\alpha_{w}$ & Constant & Constant & Variable & Variable \\
\hline
\end{tabular}

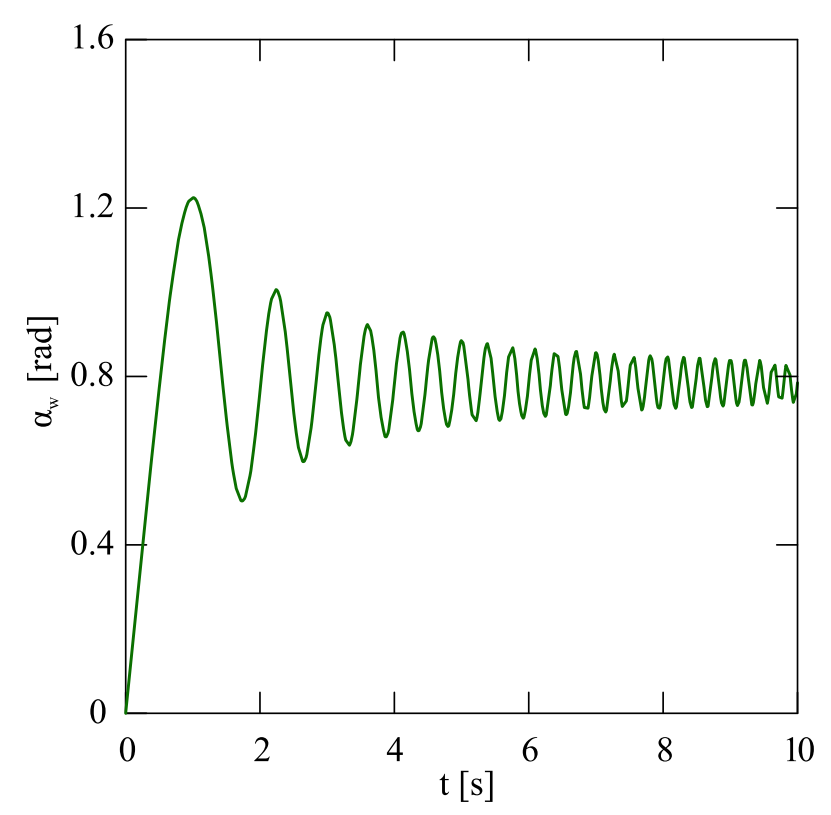

Fig. 2 Variability of wind pressure direction $\alpha_{w}(t)$ adopted in simulation tests

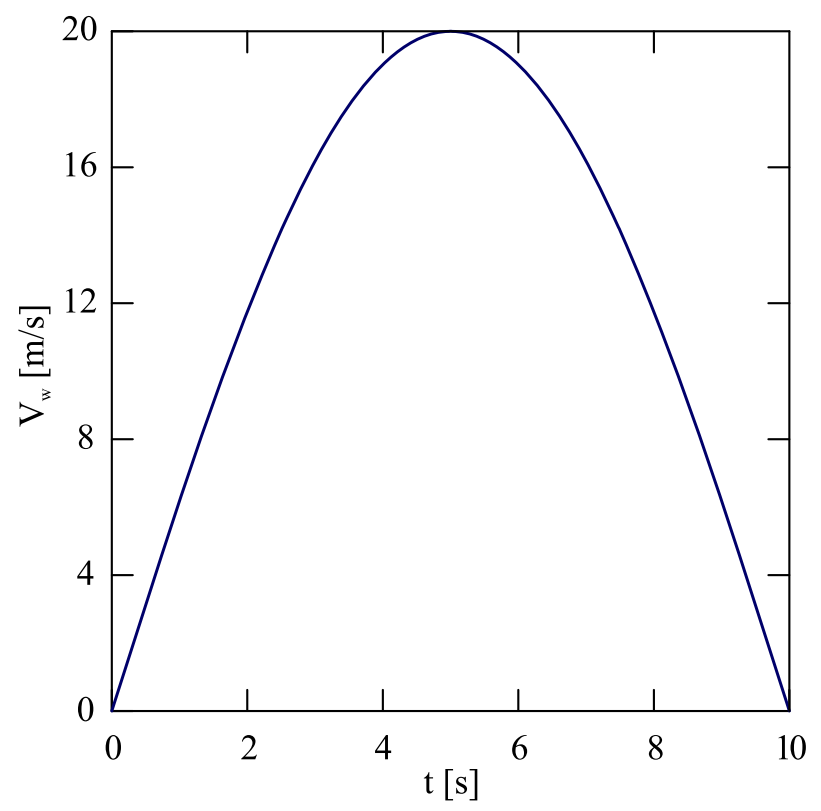

Fig. 3 Wind variability $V_{w}(t)$ adopted in simulation tests 


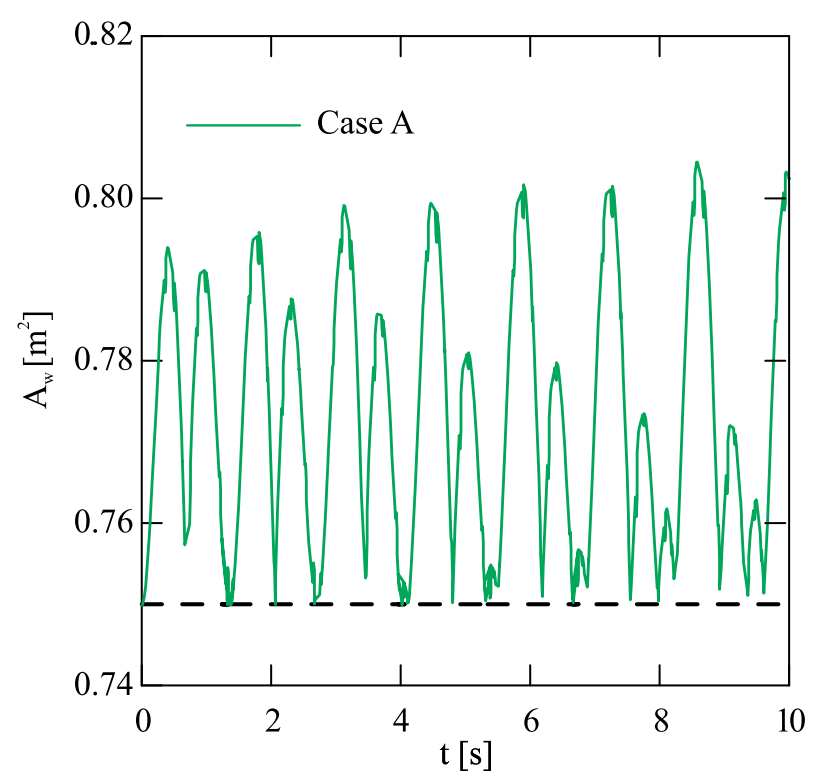

Fig. 4 Time function of effective surface variability for I variant case A (dashed line-constant area)

- rigid body mass: $m=500 \mathrm{~kg}$,

- the length of the rope: $r=2 \mathrm{~m}$,

- rigid body dimensions: $a_{1}=0.5 \mathrm{~m}, a_{2}=0.75 \mathrm{~m}, a_{3}=1.0 \mathrm{~m}$,

- total time of simulations: $t_{c}=10 \mathrm{~s}$,

- maximum wind speed: $V_{\max }=20 \mathrm{~m} / \mathrm{s}$,

- maximum value of wind angle direction: $\alpha_{\max }=\frac{\pi}{2}$.

For the simulation cases presented in Table 1, a constant value of $V_{w}$ means that the maximum wind speed is introduced, which is $20 \mathrm{~m} / \mathrm{s}$ during the entire calculation time. For the variable value of $V_{w}$, wind speed is calculated according to formula 22. The constant value of the $\alpha_{w}$ causes that wind acts along one of the coordinate system axis ( $X$ for $\alpha_{w}=0$ ). For variable $\alpha_{w}$ value, the direction is determined using the Fresnel integral (21). Calculations were made using the Runge-Kutta fourth-order method in MATLAB environment.

In the first variant, at the initial time the rigid body hung freely and remained motionless. Its motion was caused only by the force of the wind which value and direction depended on the given case. Initial conditions for this variant were as follows:

$$
\begin{aligned}
& \left.\xi(t)\right|_{t=0}=0,\left.\quad \dot{\xi}(t)\right|_{t=0}=0,\left.\quad \eta(t)\right|_{t=0}=0, \\
& \left.\dot{\eta}(t)\right|_{t=0}=0,\left.\quad \zeta(t)\right|_{t=0}=2 \mathrm{~m},\left.\quad \dot{\zeta}(t)\right|_{t=0}=0, \\
& \left.\Psi(t)\right|_{t=0}=0,\left.\quad \dot{\Psi}(t)\right|_{t=0}=0,\left.\quad \Theta(t)\right|_{t=0}=0, \\
& \left.\dot{\Theta}(t)\right|_{t=0}=0,\left.\quad \Phi(t)\right|_{t=0}=0,\left.\quad \dot{\Phi}(t)\right|_{t=0}=0 .
\end{aligned}
$$

where value of $\zeta(t)$ resulted from the initial rope length $(r)$. The results are presented in the form of a time function of the effective surface variability (Figs. 4, 5, 6,7) and percentage of variation of effective and constant area (Fig. 8). The perpendicular surface area to the wind direction, which was equal to $0.75 \mathrm{~m}^{2}$, was assumed as a constant value of the wind surface shown in Figs. 4, 5, 6 and 7 in the form of a dashed line.

Based on the results of the numerical simulations of the first variant, one can be stated that it is permissible to adopt a constant effective surface parameter only when gust with a constant direction (case B-Fig. 5) is analysed. For this case, the differences in the wind surface parameter are negligible $\left(\Delta A_{w}=0.003 \mathrm{~m}^{2}\right)$. In other cases (A, C and D), the differences are significant and may cause incorrect determination of wind force. The largest differences were observed for case $\mathrm{D}$ when constant wind speed with the variable direction was analysed. In this case, the difference was $0.2 \mathrm{~m}^{2}$. In addition, for blast of wind with variable direction (case D-Fig. 7) a lower effective area parameter was observed when $t=1 \mathrm{~s}$. Analysing the percentage variation (Fig. 8), it can be seen that the biggest differences are caused by the variable direction of wind action. One can be stated that this parameter is the most important in determining the wind force correctly. In these cases (C 


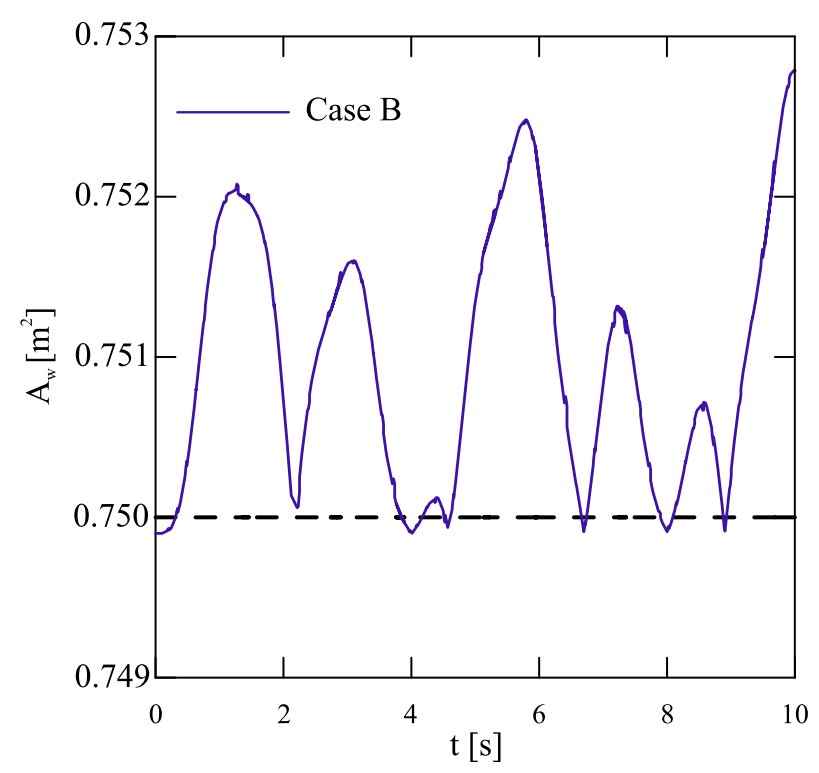

Fig. 5 Time function of effective surface variability for I variant case B (dashed line—constant area)

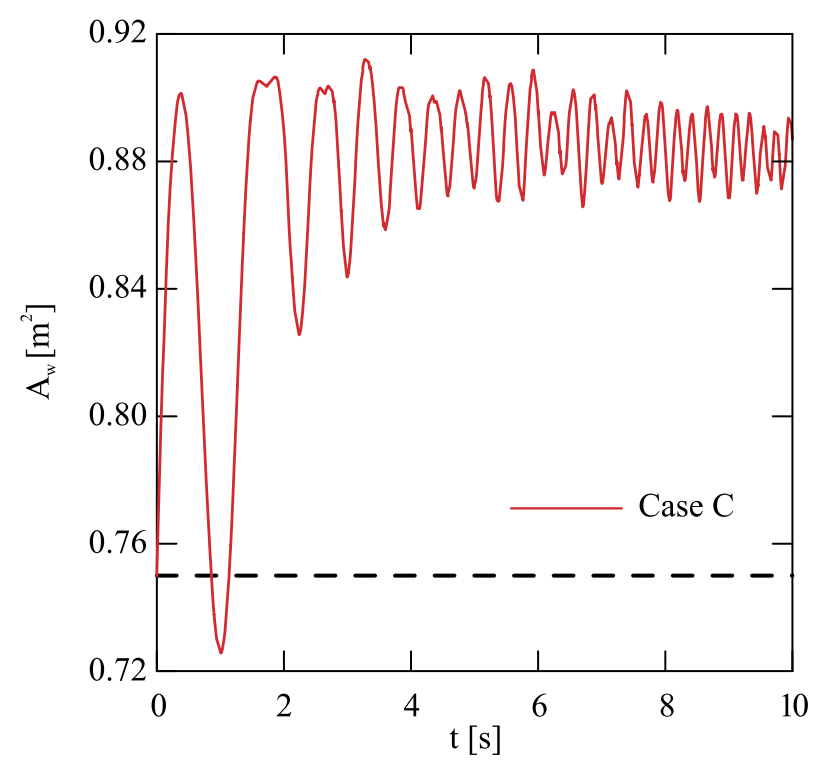

Fig. 6 Time function of effective surface variability for I variant case C (dashed line-constant area)

and D), the differences range from 20 to $25 \%$. For the case of constant wind speed and direction (A), variation did not exceed $7 \%$, while for case $\mathrm{B}$, changes are negligible.

In the second variant, the motion of the rigid body was also analysed. It was assumed that the motion would act along the X-direction. For this purpose, the following initial values were adopted:

$$
\begin{aligned}
\left.\xi(t)\right|_{t=0} & =0.5 \mathrm{~m},\left.\quad \dot{\xi}(t)\right|_{t=0}=0,\left.\quad \eta(t)\right|_{t=0}=0, \\
\left.\dot{\eta}(t)\right|_{t=0} & =0,\left.\quad \zeta(t)\right|_{t=0}=1.93649 \mathrm{~m},\left.\quad \dot{\zeta}(t)\right|_{t=0}=0, \\
\left.\Psi(t)\right|_{t=0} & =0,\left.\quad \dot{\Psi}(t)\right|_{t=0}=0,\left.\quad \Theta(t)\right|_{t=0}=0.25268 \mathrm{rad}, \\
\left.\dot{\Theta}(t)\right|_{t=0} & =0,\left.\quad \Phi(t)\right|_{t=0}=0,\left.\quad \dot{\Phi}(t)\right|_{t=0}=0 .
\end{aligned}
$$

where value of $\zeta(t)$ was calculated from algebraic relations $\left(\xi^{2}+\eta^{2}+\zeta^{2}=r^{2}\right)$. The results are shown in the form of a time function graph of the effective surface changes (Figs. 9, 10 11, 12) and percentage of variation 


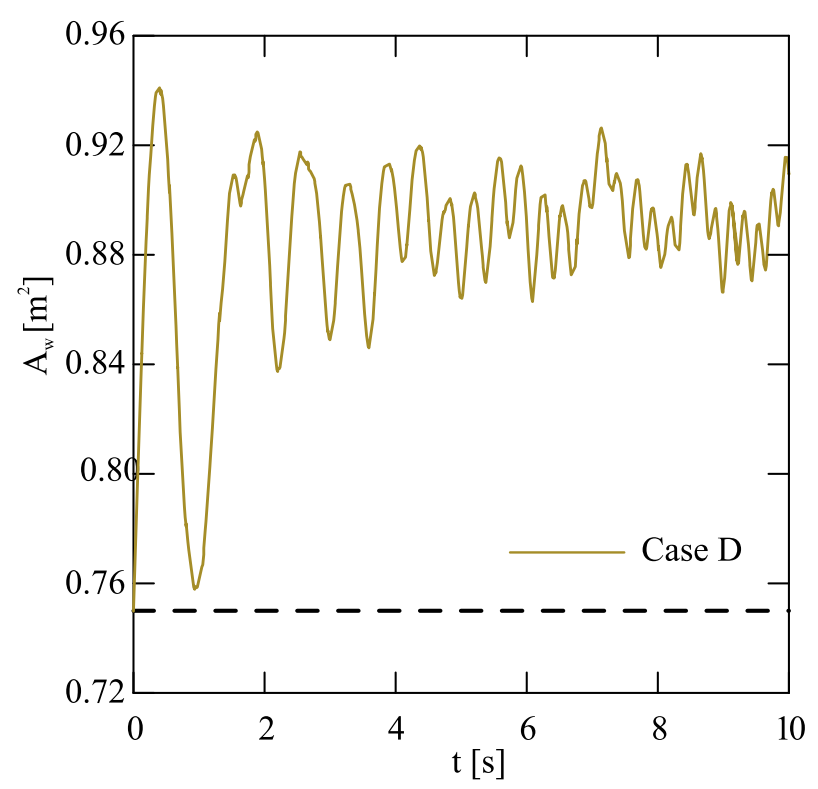

Fig. 7 Time function of effective surface variability for I variant case D (dashed line—constant area)

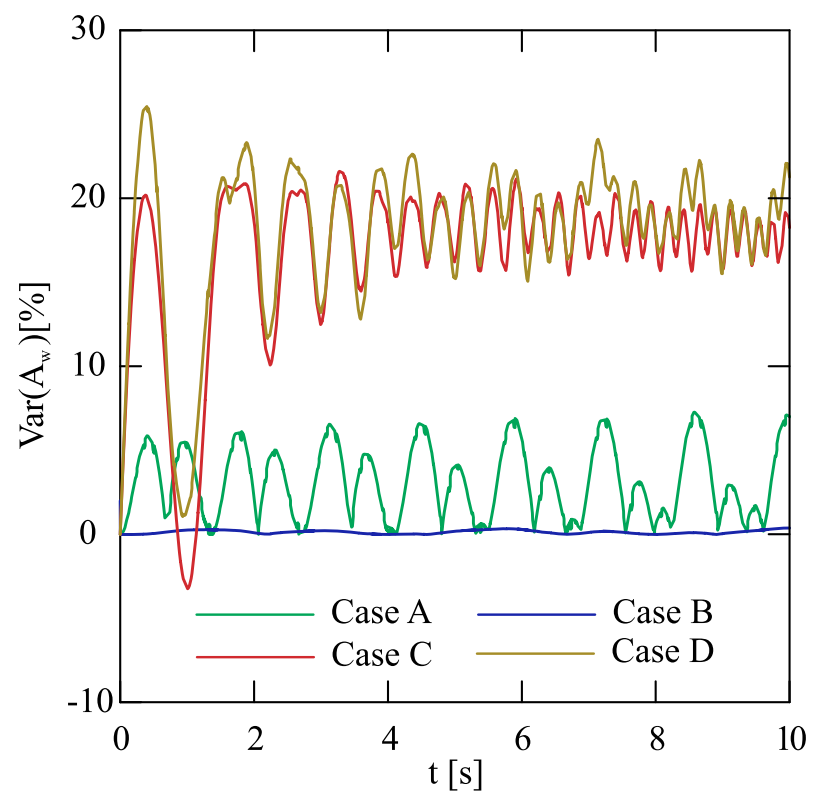

Fig. 8 Percentage of variation of variable wind area $\left(A_{w}\right)$ and constant area for I variant

of effective and constant area (Figs. 13, 14). The constant value of the effective surface was adopted as in the first variant. The projected value was read from the initial position of the body and did not change over time. It was equal to $0.82 \mathrm{~m}^{2}$. Based on the results of the second variant, it can be concluded that for the forced motion of the rigid body it is necessary to include the variable parameter of the effective surface during calculations. For the first two cases (A and B), which did not take into account the change of wind direction, the effective wind surface was in the range between the parameters of constant $\left(0.75 \mathrm{~m}^{2}\right)$ and projected $\left(0.82 \mathrm{~m}^{2}\right)$ area (Figs. 9, 10). For such cases, an average value of the surface can be determined for wind force calculations; however, it may cause incorrect determination of the rigid body behaviour and orientation. In cases where the variable direction of the wind was analysed (Figs. 11, 12), the variable surface parameter should be considered in the simulation model. For these cases, the largest differences between the projected area were observed, which were $0.13 \mathrm{~m}^{2}$ for case C and $0.18 \mathrm{~m}^{2}$ for case D. In the last case, the initial increase in effective area 


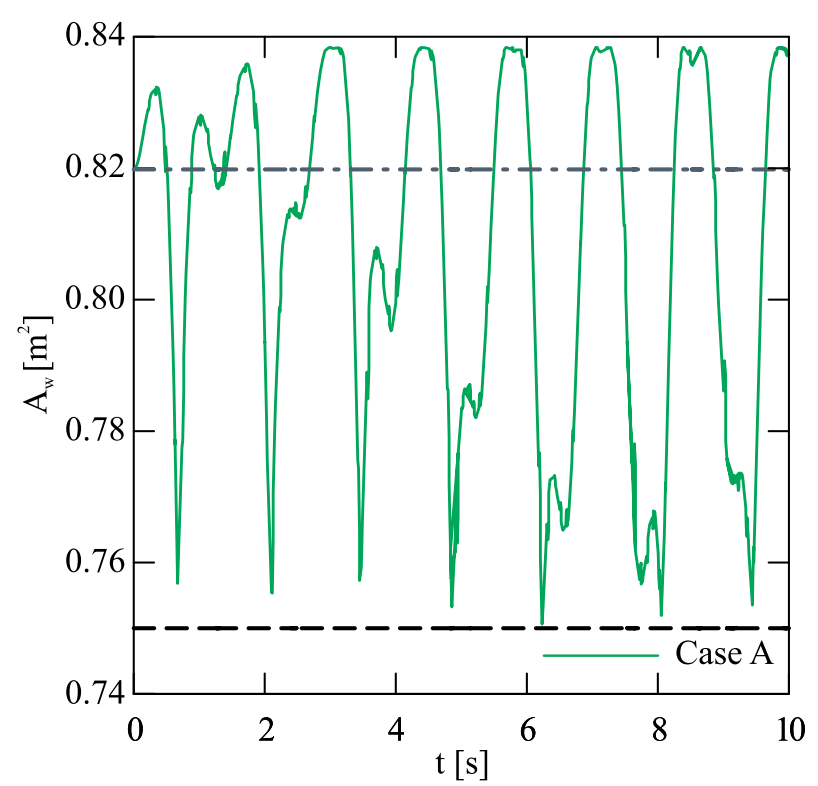

Fig. 9 Time function of effective surface variability for II variant case A (dashed line—constant area, dotted line—projected area)

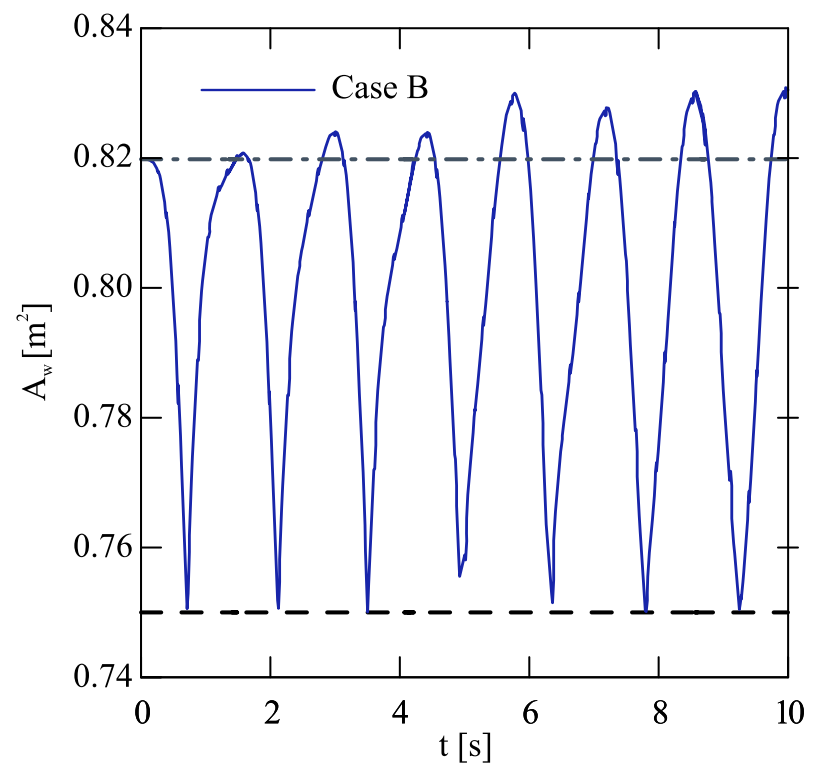

Fig. 10 Time function of effective surface variability for II variant case B (dashed line—constant area, dotted line-projected area)

and its decrease after $t=7 \mathrm{~s}$ were also observed. This is due to the behaviour of the body, which under the influence of the maximum of wind swing, significantly reduces the surface area. At this point, despite the constant value of wind speed, the wind force was the smallest, which caused the body to return to its previous position and increase in the effective area. Comparing the results of percentage variations (Figs. 13, 14), it can be seen that adopting a constant area parameter $\left(A_{w}=0.75 \mathrm{~m}^{2}\right)$ for forced motion causes larger differences than the projected area $\left(A_{w}=0.82 \mathrm{~m}^{2}\right)$. Regardless of the parameter used (constant or projected), the absolute variation value reaches $10 \%$. The largest differences can be seen in the case where the wind speed is constant, but its direction changes (case D). In this case, the percentage variation of the constant surface area (Fig. 13) was in the range $<-28 \%, 30 \%>$. For a gust with a variable direction, the maximum absolute difference was $29 \%$. For the projected surface area parameter (Fig. 14), the percentage variation was in the range $<-33 \%$, $19 \%>$. For case $\mathrm{C}$, the maximum absolute value was $18 \%$. 


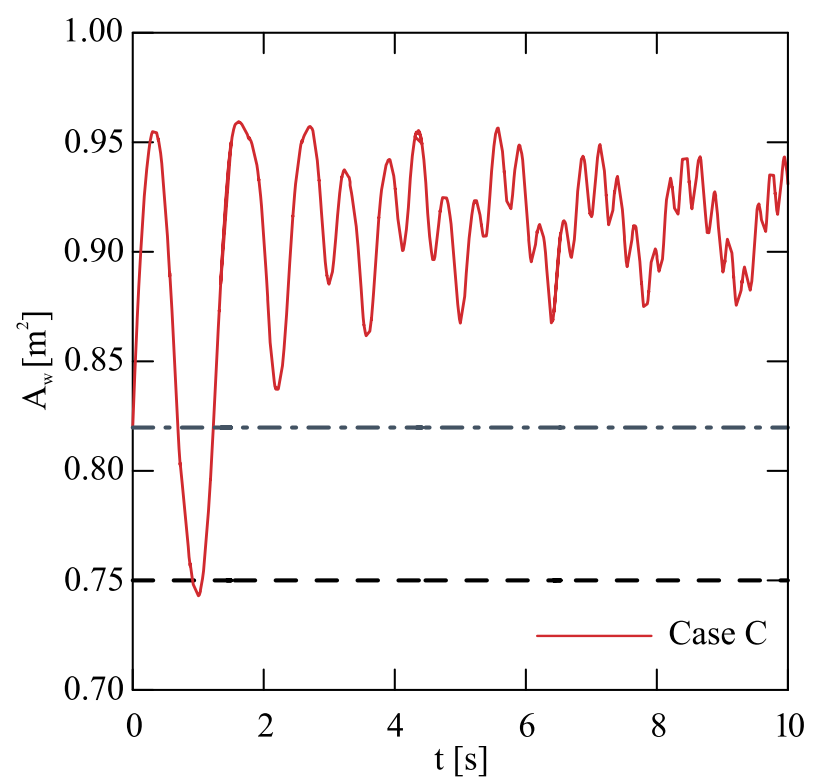

Fig. 11 Time function of effective surface variability for II variant case C (dashed line—constant area, dotted line-projected area)

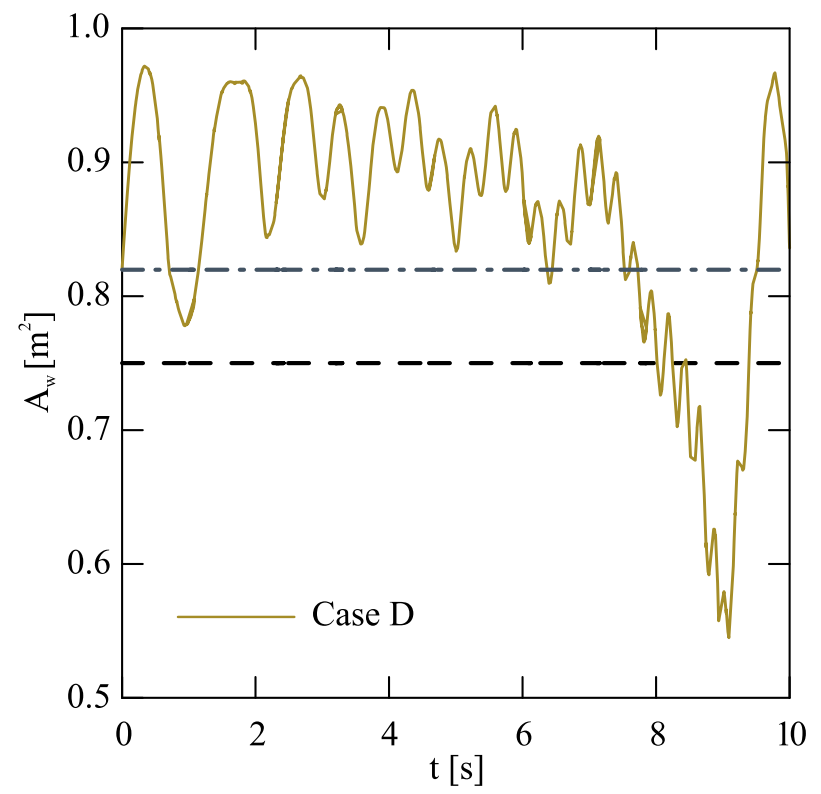

Fig. 12 Time function of effective surface variability for II variant case D (dashed line—constant area, dotted line—projected area)

\section{Conclusions}

The paper proposes a new method of determining the wind area of a rigid body under wind disturbances. The effective surface area parameter is used to determine the current wind force and body orientation in space. The theoretical model takes into account the variability of the effective surface area used to calculate wind loads. To describe the method, dependencies of normal vectors and body vertices were used. To determine the coordinate vertices, Cardan angles were taken into account. These angles were determined on the basis of the cosine matrix of elementary rotation as well as the dependence of angular velocity and acceleration. The general motion of the rigid body is presented as two vector equations describing body translation and rotation. A system of six second-order differential equations was obtained that describe the initial problem of 


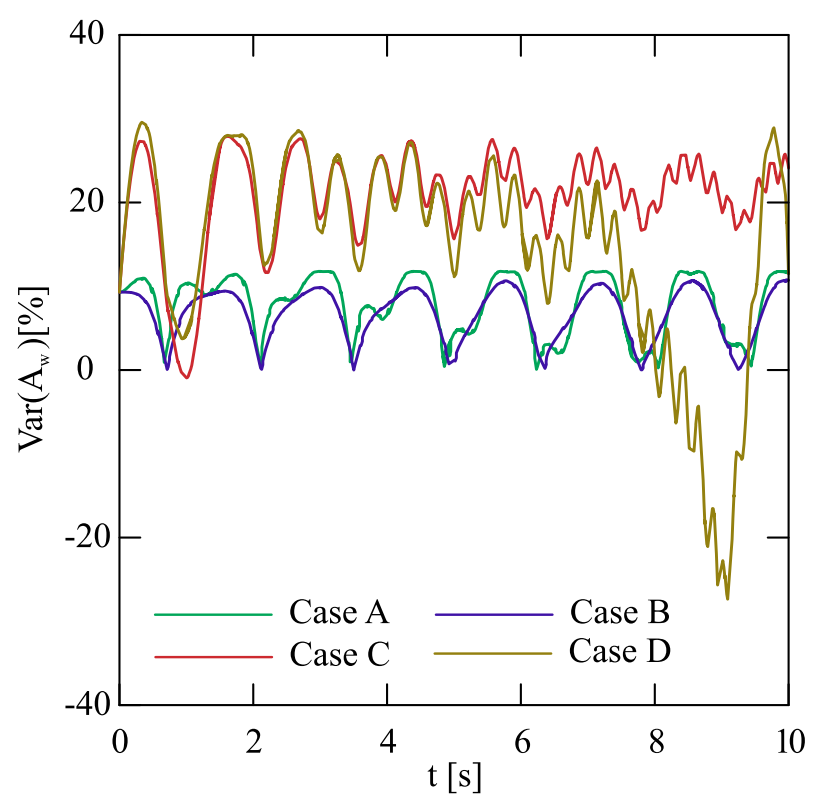

Fig. 13 Percentage of variation of variable wind area $\left(A_{w}\right)$ and constant area for II variant

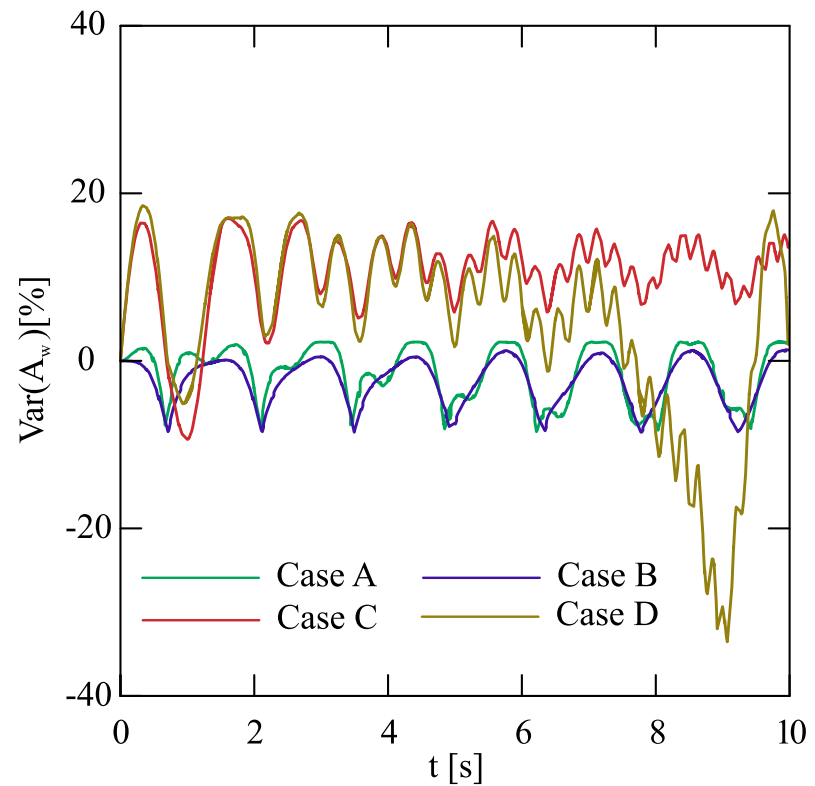

Fig. 14 Percentage of variation of variable wind area $\left(A_{w}\right)$ and projected area for II variant

the motion. The problem formulated in this work was solved using the Runge-Kutta fourth method. Numerical calculations were carried out in MATLAB software.

The results of the example numerical simulations are presented for two variants. In the first, the problem was analysed when the rigid body hangs freely in the initial state of calculations. Its motion is caused only under the influence of wind. The second variant concerned also the forced motion of a rigid body in one plane. For both variants, four cases were analysed differing in wind speed and direction of its impact. To model the variability of the wind force direction, the Fresnel cosine integral was used. For mapping the gust, the maximum wind speed was defined and the course of sine law was used. The obtained effective surface values were compared with constant (I and II variant) and projected (II variant) values. For the first variant, the smallest differences were obtained for case $\mathrm{B}$, in which the blast of wind was analysed without change in its direction. The largest changes were noted for cases C and D. For the second variant, cases A and B 
presented similar variations, while the larger differences were noted for constant speed and variable direction of the wind.

Based on the results obtained, it can be concluded that depending on the analysed problem, the wind area parameter should be selected appropriately. If the problem of body motion is analysed only under the influence of gusts without changes in their direction, it is permissible to use a constant surface area. In the case when the forced motion of solid is analysed at constant and variable wind speed, it is possible to specify the effective surface area parameter as the projected average. However, when analysing the variability of wind direction, it is necessary to include the variable surface area parameter in the theoretical model. It should be noted that any approximation of the wind area will cause differences in the behaviour of the body. In order to accurately determine, for example the positioning of such a solid, the proposed method should be used.

The model presented in this work can be used during research on the transport of loads carried by any device equipped with a rope system. After taking into account the relevant control functions, it can also be a tool for visualizing the load positioning under the wind disturbances. This work will be further developed, among others, by taking into account the rope system deformation, shape variability of body and other wind loads issues (e.g. von Karman vortices).

Acknowledgements This work has been carried out within statutory research of the Department of Mechanics and Machine Design Fundamentals of Czestochowa University of Technology.

Open Access This article is licensed under a Creative Commons Attribution 4.0 International License, which permits use, sharing, adaptation, distribution and reproduction in any medium or format, as long as you give appropriate credit to the original author(s) and the source, provide a link to the Creative Commons licence, and indicate if changes were made. The images or other third party material in this article are included in the article's Creative Commons licence, unless indicated otherwise in a credit line to the material. If material is not included in the article's Creative Commons licence and your intended use is not permitted by statutory regulation or exceeds the permitted use, you will need to obtain permission directly from the copyright holder. To view a copy of this licence, visit http://creativecommons.org/licenses/by/4.0/.

\section{Compliance with ethical standards}

Conflict of interest The authors declare that they have no conflict of interest.

\section{References}

1. Andersen, I.M.V.: Wind loads on post-panamax container ship. Ocean Eng. 58, 115-134 (2013). https://doi.org/10.1016/j. oceaneng.2012.10.008

2. Bracke, X., de Vyver, J.V., Kooning, J.D., Vandevelde, L.: Effective capture of wind gusts in small wind turbines by using a full active rectifier. In: 3rd Renewable Power Generation Conference (RPG 2014). Institution of Engineering and Technology (2014). https://doi.org/10.1049/cp.2014.0835

3. Canuto, E., Novara, C., Massotti, L., Carlucci, D., Montenegro, C.P.: Spacecraft Dynamics and Control. Elsevier, Amsterdam (2018). https://doi.org/10.1016/c2016-0-00420-5

4. Cekus, D., Kwiatoń, P.: Analysis of the motion of the load carried by a laboratory mobile crane. In: Engineering Mechanics 2018. Institute of Theoretical and Applied Mechanics of the Czech Academy of Sciences (2018). https://doi.org/10.21495/ 91-8-137

5. Cekus, D., Kwiaton, P.: Effect of the rope system deformation on theworking cycle of the mobile crane during interaction of windpressure. Mech. Mach. Theory 153, 104011 (2020). https://doi.org/10.1016/j.mechmachtheory.2020.104011

6. Cekus, D., Gnatowska, R., Kwiaton, P.: Impact of wind on the movement of the load carried by rotary crane. Appl. Sci. 9(18), 3842 (2019). https://doi.org/10.3390/app9183842

7. Cekus, D., Gnatowska, R., Kwiatoń, P., Šofer, M.: Simulation research of a wind turbine using SolidWorks software. J. Phys. Conf. Ser. 1398, 012001 (2019). https://doi.org/10.1088/1742-6596/1398/1/012001

8. Chung, P.H., Chou, C.C., Yang, R.Y., Chung, C.Y.: Wind loads on a PV array. Appl. Sci. 9(12), 2466 (2019). https://doi.org/ 10.3390/app9122466

9. Curtis, H.D.: Orbital Mechanics for Engineering Students. Elsevier, Amsterdam (2020). https://doi.org/10.1016/c2016-002107-1

10. Guo, J., Zhu, M., Hu, C.: Study on wind load shape factor of long-span stadium roof. Adv. Struct. Eng. (2020). https://doi. org/10.1177/1369433220908111

11. Hua, C., Chen, J., Guan, X.: Adaptive prescribed performance control of QUAVs with unknown time-varying payload and wind gust disturbance. J. Frankl. Inst. 355(14), 6323-6338 (2018). https://doi.org/10.1016/j.jfranklin.2018.05.062

12. Huang, J., Maleki, E., Singhose, W.: Dynamics and swing control of mobile boom cranes subject to wind disturbances. IET Control Theory Appl. 7(9), 1187-1195 (2013). https://doi.org/10.1049/iet-cta.2012.0957

13. ISO 4302:2016 Cranes-wind load assessment 
14. Jaskot, A., Posiadała, B.: Model of dynamics of the three wheeled mobile platform. In: Kacprzyk, J. (ed.) Advances in Intelligent Systems and Computing, pp. 184-191. Springer, New York (2019). https://doi.org/10.1007/978-3-030-158576_19

15. Jiang, H., Li, Y.: Dynamic reliability analysis of tower crane with wind loading. IOP Conf. Ser. Mater. Sci. Eng. 677, 052031 (2019). https://doi.org/10.1088/1757-899x/677/5/052031

16. Kwiatoń, P., Kubik, I.: Usage of Bryant angles to load movementanalysis. In: Czyż, Z. (ed.) Contemporary Problems of Material Engineering and Mechanics, pp. 214-227. Tygiel Scientific Publisher (2018) (in Polish)

17. Majidian, H., Azarsina, F.: Aerodynamic simulation of a containership to evaluate cargo configuration effect on frontal wind loads. China Ocean Eng. 32(2), 196-205 (2018). https://doi.org/10.1007/s13344-018-0021-1

18. Moon, F.C.: Applied Dynamics: With Applications to Multibody and Mechatronic Systems. Wiley, New York (2008)

19. Posiadala, B., Warys, P., Cekus, D., Tomala, M.: The dynamics of the forest crane during the load carrying. Int. J. Struct. Stab. Dyn. 13(07), 1340013 (2013). https://doi.org/10.1142/s0219455413400130

20. Sandoval-Hernandez, M., Vazquez-Leal, H., Hernandez-Martinez, L., Filobello-Nino, U.A., Jimenez-Fernandez, V.M., Herrera-May, A.L., Castaneda-Sheissa, R., Ambrosio-Lazaro, R.C., Diaz-Arango, G.: Approximation of fresnel integrals with applications to diffraction problems. Math. Probl. Eng. 2018, 1-13 (2018). https://doi.org/10.1155/2018/4031793

21. Schneider, P.J., Eberly, D.H.: Geometric primitives in 3D. In: Geometric Tools for Computer Graphics, pp. 325-364. Elsevier (2003). https://doi.org/10.1016/b978-155860594-7/50012-6

22. Singh, J., Roy, A.K.: Effects of roof slope and wind direction on wind pressure distribution on the roof of a square plan pyramidal low-rise building using CFD simulation. Int. J. Adv. Struct. Eng. 11(2), 231-254 (2019). https://doi.org/10.1007/ s40091-019-0227-3

23. Wu, C.H., Kopp, G.A.: Estimation of wind-induced pressures on a low-rise building using quasi-steady theory. Front. Built Environ. 2, 10 (2016). https://doi.org/10.3389/fbuil.2016.00005

24. Żelazny, K.: Approximate method of calculation of the wind action on a bulk carrier. Sci. J. Marit. Univ. Szczecin 38(110), 131-135 (2014)

Publisher's Note Springer Nature remains neutral with regard to jurisdictional claims in published maps and institutional affiliations. 